\title{
Centro de Pesquisa de História Natural e Arqueologia do Maranhão
}

Deusdédit Carneiro Leite Filho

Arqueólogo-diretor, chefe do Centro de Pesquisa de História Natural e Arqueologia do Maranhão.

E-mail: decalef@ig.com.br

Resumo: O Centro de Pesquisa de História Natural e Arqueologia do Maranhão - CPHNAMA, vinculado à Secretaria de Cultura do Estado, tem como objetivo promover o estudo, a valorização e a preservação do acervo patrimonial maranhense, especificamente os recursos e bens arqueológicos, paleontológicos e a cultura material e tradições dos povos indígenas no Maranhão. Além do desenvolvimento sistemático de atividades de pesquisa nas áreas de Paleontologia, Arqueologia e Etnologia, desenvolve ações voltadas à educação patrimonial através da elaboração, organização e execução de exposições, eventos culturais, mostras, seminários, palestras e aulas direcionadas a públicos diferenciados.

Palavras-chave: paleontologia, arqueologia, etnologia, museu, Maranhão.
Abstract: The Research Center of $\mathrm{Na}$ tural History and Archaeology of Maranhão - CPHNAMA, linked to the State Secretary of Culture, has as objective to promote the study, valorization and preservation of Maranhão patrimonial heritage, specifically the archaeological and palaeontological resources and assets and the material culture and traditions of indigenous peoples. Besides the systematic development of research activities in the areas of Paleontology, Archaeology and Ethnology, it develops patrimonial education-focused actions through the preparation, organization and execution of exhibitions, cultural events, exhibitions, seminars, lectures and classes for different audiences.

Keywords: paleontology, archeology, ethnology, museum, Maranhão.

Fundado em março de 2002, o Centro de Pesquisa de História Natural e Arqueologia do Maranhão - CPHNAMA tem como objetivo o estudo, a valorização e a preservação do acervo patrimonial maranhense, especificamente os recursos e bens arqueológicos, paleontológicos e a cultura material e tradições dos povos indígenas no Maranhão. Com vínculo na Secretaria de Cultura do Estado, desenvolve projetos de pesquisas sistemáticas em parceria com instituições regionais e nacionais, planejando e executando ações paralelas voltadas à educação patrimonial através da orientação de atividades culturais em escolas 
comunicação \& educação • Ano XV • número 2 • maio/ago 2010

das redes pública e particular e realizando palestras em universidades e eventos regionais. A divulgação do potencial do estado nas áreas em questão visa à sensibilização e conscientização dos diferentes públicos visitantes acerca da necessidade de uma gestão compartilhada desses recursos, uma vez que a preservação da nossa herança cultural cabe a todos.

\section{OS ESPAÇOS}

O CPHNAMA está sediado em um sobrado colonial localizado na Praia Grande, centro Histórico de São Luís. O edifício foi construído pelo Capitão Antonio José de Souza em 1800 e sediou, entre outras ocupações, a tipografia e redação do diário republicano $O$ Globo, editado por Francisco de Paula Belfort Duarte, assim como na década de 1980 o Projeto Reviver, programa de revitalização do conjunto de imóveis históricos situados na Praia Grande, que transformou o local em ponto de referência cultural.

Atualmente, a instituição abriga em seus dois andares as instalações do Museu. A instituição oferece à comunidade visitação guiada gratuita a três exposições, organizadas em salas temáticas.

Em 2009, o CPHNAMA atendeu cerca de 14 mil visitantes, a maioria estudantes de escolas públicas, particulares e universitários.

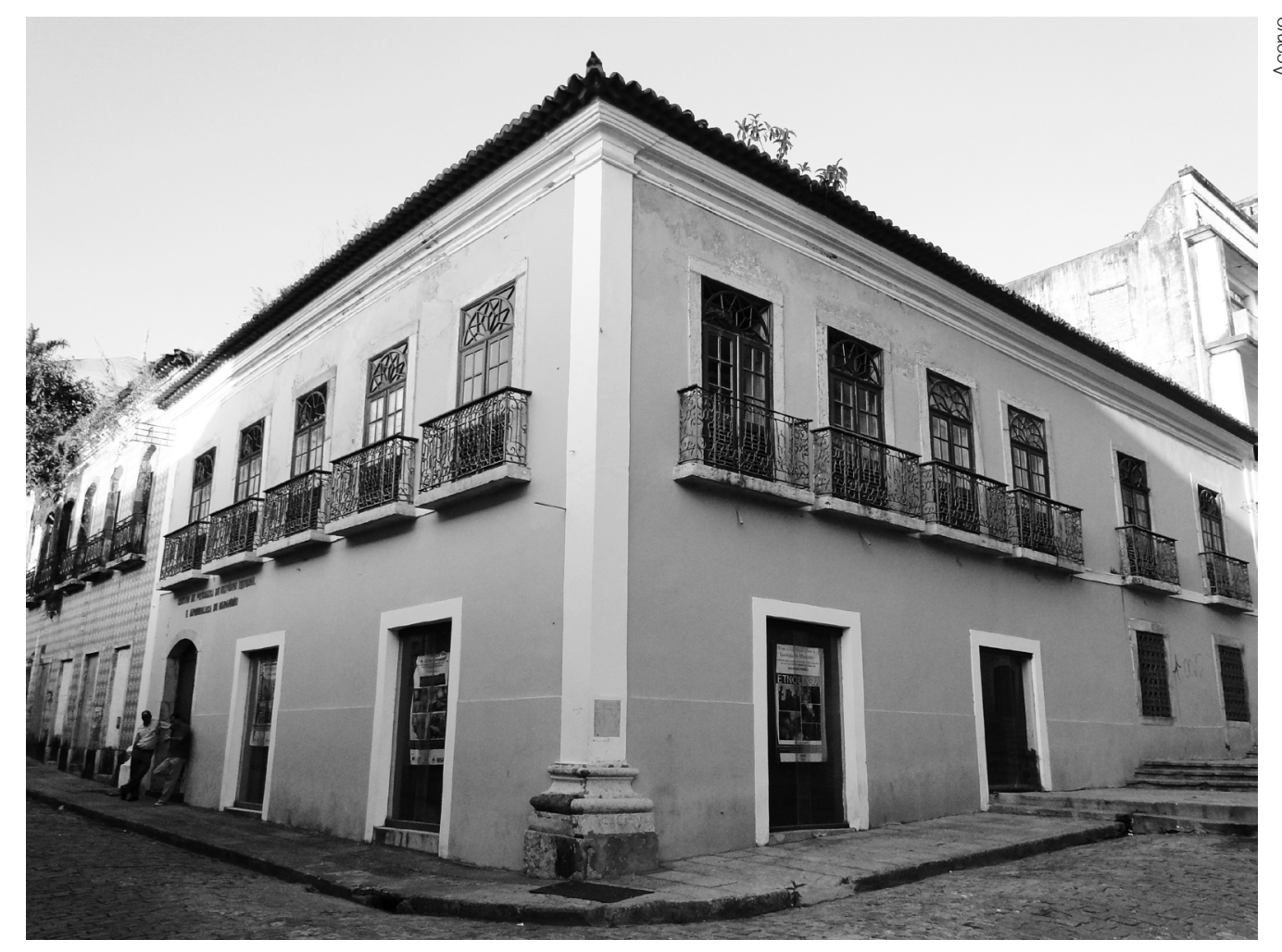

Sobrado colonial localizado na Praia Grande, centro Histórico de São Luís e sede do CPHNAMA. 
Centro de Pesquisa de História Natural e Arqueologia • Deusdédit C. L. Filho

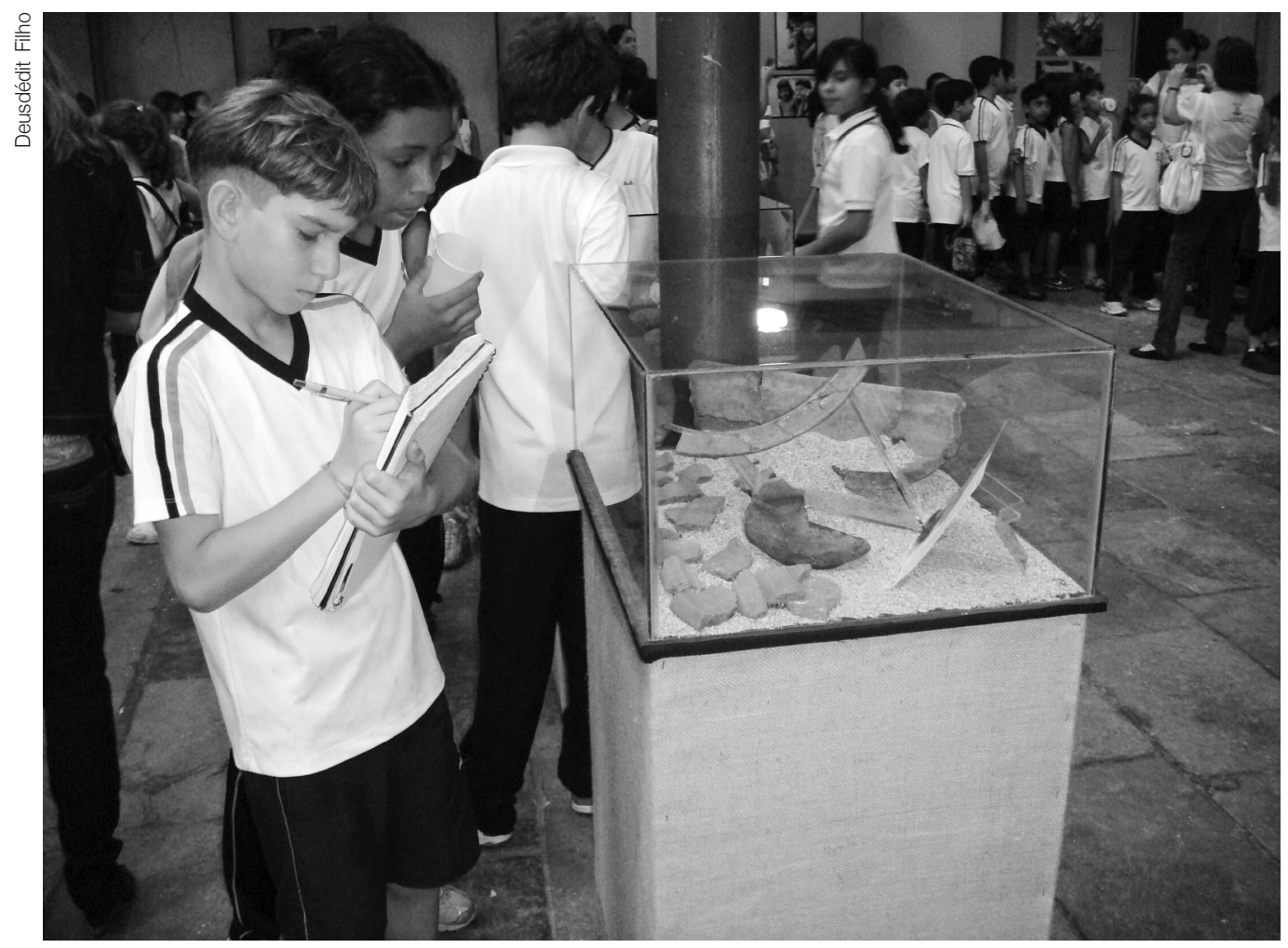

Exposição arqueológica na Semana dos Povos Indígenas. Acervo: Centro de Pesquisa.

A exposição paleontológica apresenta fósseis de animais e plantas encontrados no Maranhão e réplicas, em escala reduzida, de espécies da megafauna que existiram há cerca de 100 milhões de anos. O acervo arqueológico compreende artefatos utilitários, cerimoniais e de uso pessoal provenientes da presença e atividade humana no Maranhão, abrangendo desde o processo de ocupação dos primeiros grupos pré-coloniais, o período posterior de chegada e fixação do colonizador, até o período histórico subsequente.

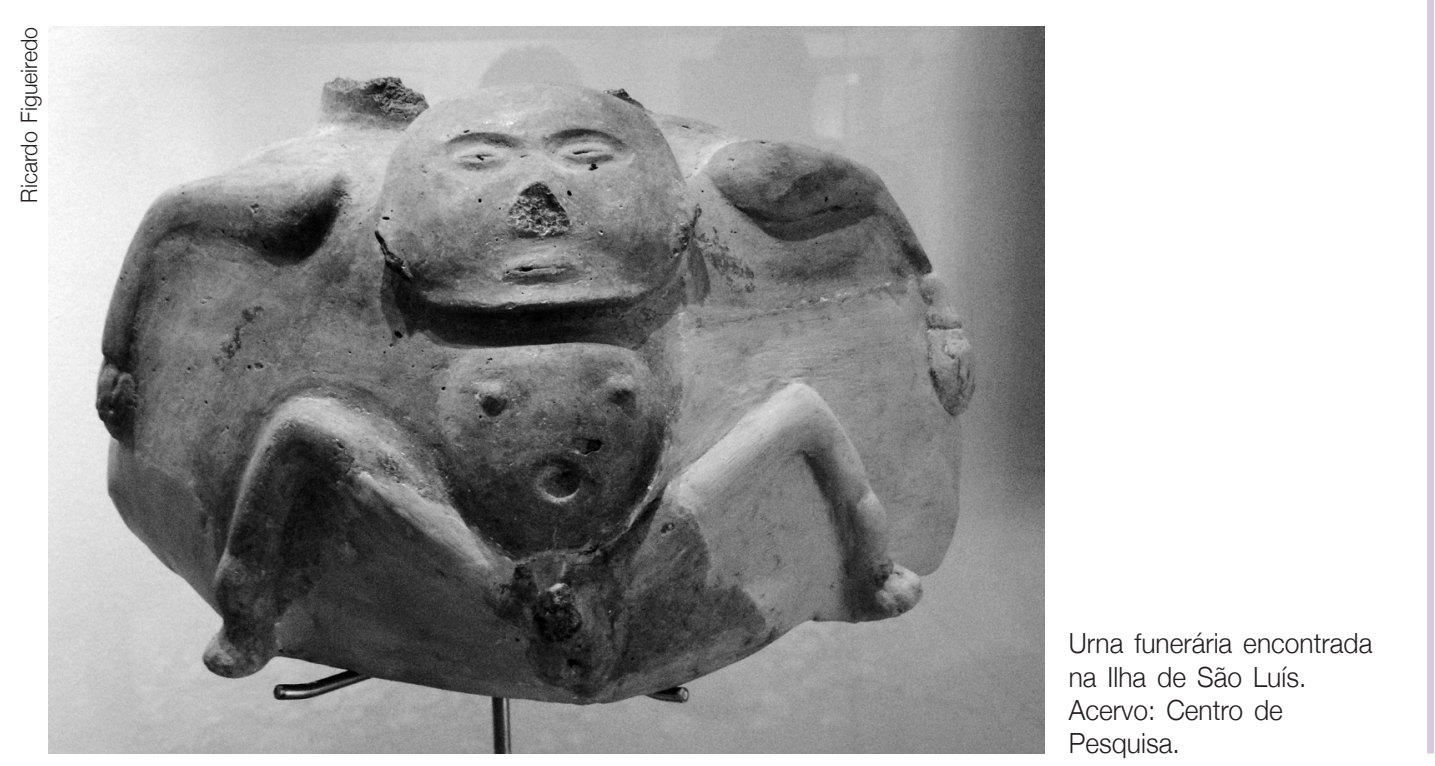


O espaço dedicado à etnologia tem em exposição objetos manufaturados pelos povos indígenas no Maranhão e utilizados por grupos Guajajara-Tenetehara, Canela, Krikati, Urubu-Ka’apor, Awá-Guajá, Timbira e Gavião, em seus diversos rituais e atividades cotidianas.

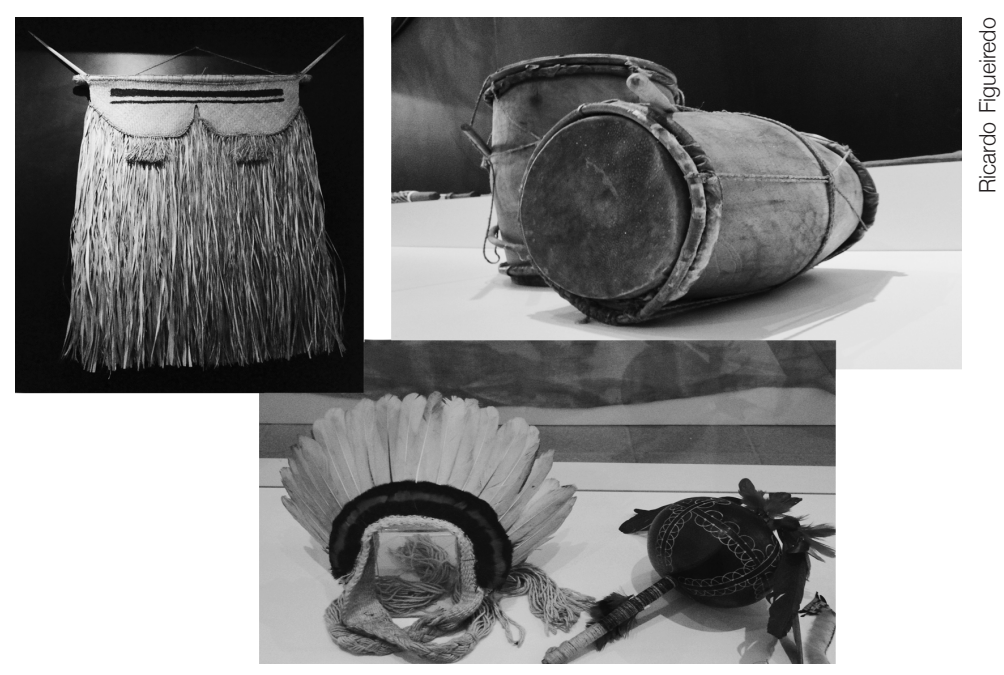

Máscara ritualística Canela-Ma, cocar e maracá Kaapor e tambores cerimoniais Kaapor. Acervo: Centro de Pesquisa.

\section{Sala expositiva de Paleontologia}

Abriga fósseis e réplicas de espécies pré-históricas encontradas no Maranhão. Recentemente, foi encontrado na Ilha do Cajual um novo tipo de material fóssil até então desconhecido para os paleontólogos do Maranhão. O material consiste em pequenas vértebras associadas a uma serpente de 105 milhões de anos.

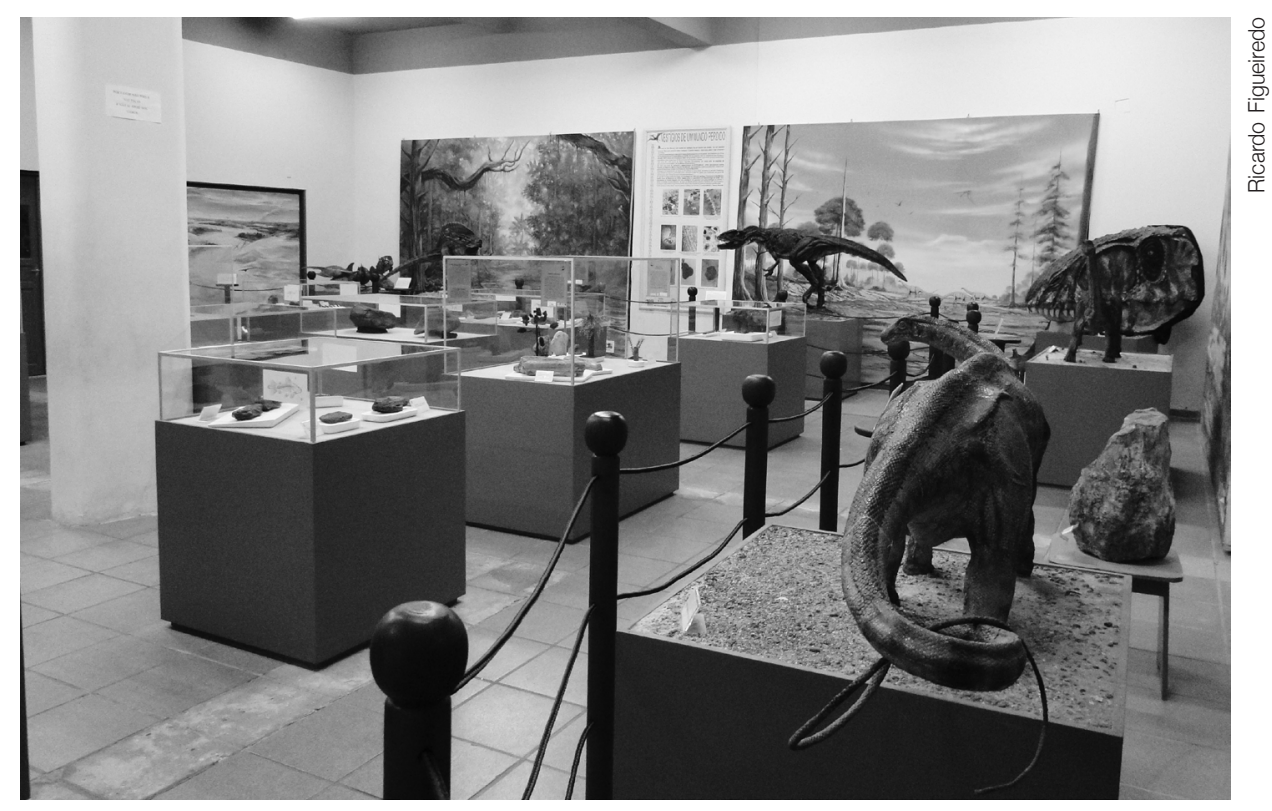

Exposição de Paleontologia. 
Centro de Pesquisa de História Natural e Arqueologia • Deusdédit C. L. Filho

\section{Sala expositiva de Arqueologia}

São expostos artefatos de pedra, objetos cerâmicos utilitários e ritualísticos pré-coloniais, utensílios de louça e artigos de uso pessoal e cotidiano provenientes do período histórico. A iluminação da exposição do setor de Arqueologia foi modernizada para atender às necessidades funcionais e estéticas do espaço, como também para oferecer maior conforto visual aos frequentadores e eventuais visitantes.

\section{Sala expositiva de Etnologia}

O visitante pode apreciar objetos de uso diário e de cerimoniais pertencentes aos grupos indígenas contemporâneos. Abriga também a Biblioteca Olavo Correia Lima - com acesso à consulta ao seu acervo de cerca de 3 mil volumes nas áreas de atuação do órgão.

\section{FORMAS DE ATUAÇÃO}

Além do desenvolvimento sistemático de atividades de pesquisa nas áreas de Paleontologia, Arqueologia e Etnologia, o CPHNAMA desenvolve ações voltadas à educação patrimonial através da elaboração, organização e execução de exposições, eventos culturais, mostras, seminários, palestras e aulas direcionadas a públicos diferenciados.

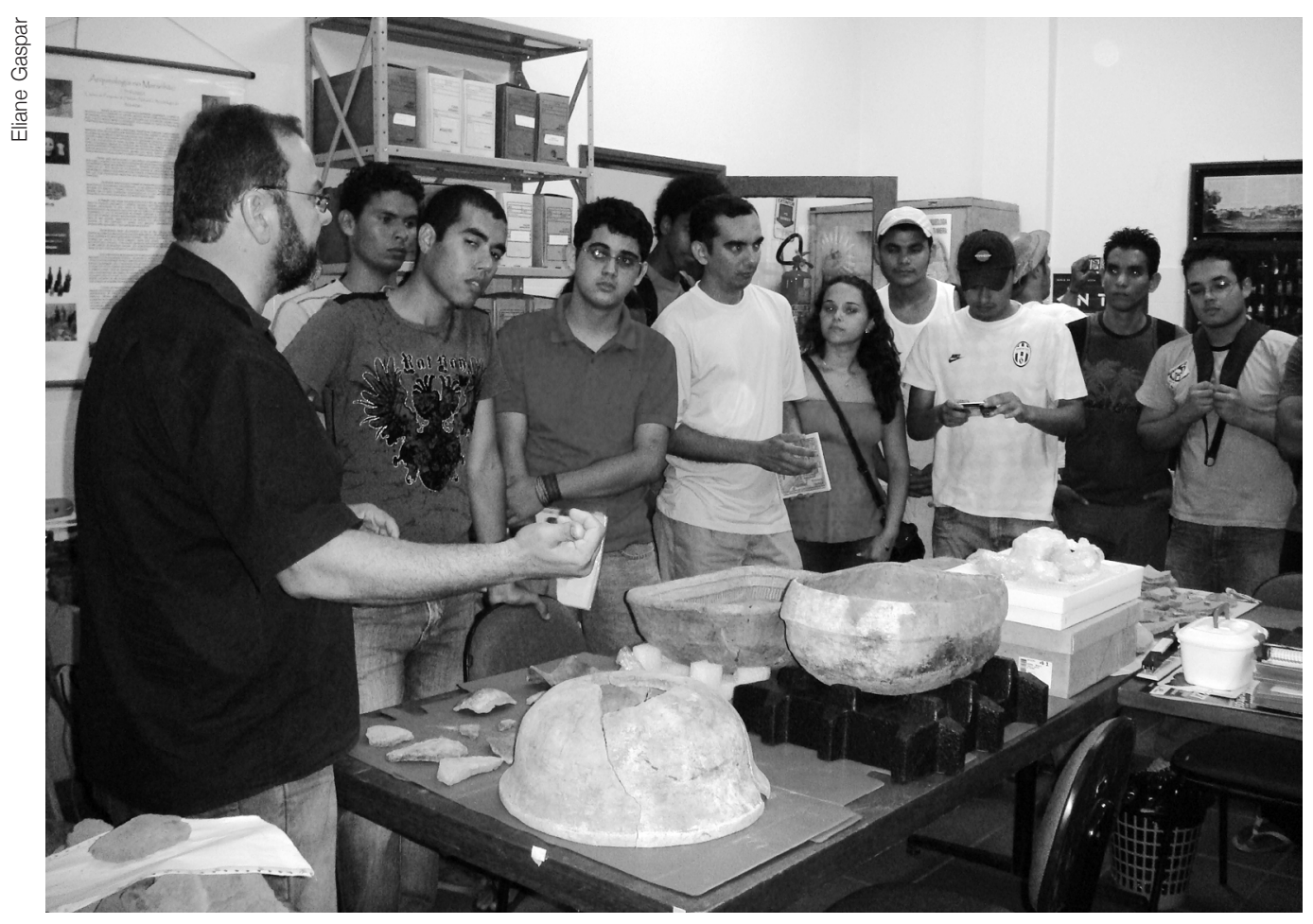

Visita técnica dos alunos do curso de História da UFMA ao laboratório de Arqueologia. 
comunicação \& educação • Ano XV • número 2 • maio/ago 2010

\section{PUBLICAÇÕES}

Para oferecer apoio à visitação da exposição arqueológica da instituição, foi lançado o livro Arqueologia no Maranhão. A cartilha está sendo distribuída como veículo de divulgação junto a escolas, professores, visitantes e ao público frequentador das exposições temáticas. O trabalho foi concebido pelos arqueólogos Deusdédit Leite e Eliane Gaspar, que assinam também os textos explicativos sobre o período pré-colonial maranhense (arte rupestre nos abrigos sob rocha, os sambaquis, as estearias, as populações de horticultores e ceramistas, as nações indígenas encontradas pelos colonizadores) e o metódico trabalho do arqueólogo. Ao lado de cada texto, ilustrações de Terciano (Cartum Postal), que para representar os paleoíndios do Maranhão mudou seu traço artístico, inspirado na iconografia representativa de ameríndios das décadas passadas. Já o projeto gráfico é assinado por Henrique Dias. A ideia é divulgar para professores e alunos, de forma didática, a arqueologia produzida no Maranhão e o acervo exposto no Museu.

\section{Serviço}

\section{Centro de Pesquisa de História Natural e Arqueologia do Maranhão}

Rua do Giz, n. 59, Centro Histórico, Praia Grande, São Luís-MA - CEP: 65010-680. Horário de funcionamento: segundas a sextas-feiras, das $8 \mathrm{~h}$ às $12 \mathrm{~h}$ e das $14 \mathrm{~h}$ às $18 \mathrm{~h}$. Telefone: (98) 3218-9906.

Site: <http://www.cultura.ma.gov.br/portal/cphna/index.php>. 